\title{
The Distributed OTDR Measuring System with UWFBGs based on Correlation Degree Spectrum
}

\author{
Rong Zhou ${ }^{1, a}$ Qi Zhang ${ }^{1, b}$ \\ ${ }^{1}$ School of information Engineering, Wuhan University of Technology Wuhan, 430070, China \\ a397232719@qq.com, bzhangqi6028@sina.com.cn
}

\begin{abstract}
Keywords: UWFBGs, Correlation degree spectrum, CW noise, micro-strain, distributed Algorithm
\end{abstract} Abstract. The distributed OTDR measuring system based on correlation degree spectrum of UWFBGs is proposed. The traditional OTDR system uses a white light source to reduce the noise signal induced by the interference from multiple reflections between UWFBGs (reflectivity of each to be $0.1 \%$ ), in addition we used the optical pulse with high extinction ratio $(>40 \mathrm{~dB})$ to reduce the noise floor of OTDR trace, so that the large number (330) of the FBGs can be detected at the end of $850 \mathrm{~m}$ due to the low noise floor. The spectrums of $330 \mathrm{FBG}$ have been characterized with a high-precision tunable filter at the receiver end. By calculating the correlation degree of adjacent spectrum we improved SNR to $35 \mathrm{~dB}$. The wavelength shift accuracy is $3.2 \mathrm{pm}$. The accuracy of measured temperature and strain are respectively 3.2 and $0.32^{\circ} \mathrm{C}$.

\section{Introduction}

The distributed OTDR measuring system is widely used in the civil infrastructure, industrial mechanism monitoring, intrusion detection due to its capability of high sensitivity and distributed measurement[1-3]. Furthermore, because of the high reflection ratio contrast to the Rayleigh backscattering, the UWFBGs emerge in both the phase-OTDR and traditional OTDR measuring system[4-7]. In the OTDR system with fiber which is uniform medium, there is only Rayleigh backscattering through fiber, the inner intrinsic noise can be negligible. However, in the OTDR system with UWFBG, there are both Rayleigh backscattering and high reflection coefficient $(0.1-1 \%)$ light of UWFBGs, and the medium is not uniform (what do you mean by medium is not uniform), then the optical route difference comes into being between adjacent UWFBGs. Once the distributed phase-OTDR sensing systems with UWFBGs use narrow line-width laser[4-5], the strong reflection light can propagate between two adjacent UWFBGs to generate intrinsic interference which may cause additional noise to eliminate SNR, and the number of UWFBGs or the length of fiber can be used are all decreased. Why is your proposed technique to overcome previous work limitation? What is the principle of your proposed technique to give better result?. That's why the distributed traditional OTDR sensing systems with UWFBGs mostly use broad band laser[6-7], and the number of UWFBGs or the length of fiber is much more than that in phase-OTDR system. What is the reason of the broadband source better than narrow band source for weak FBG comparing with optical fibers, which shows better result for phase OTDR rather than OTDR?

To the existing phase-OTDR measuring system with UWFBGs, the temperature can be measure with the resolution of $0.42 \mathrm{~K}$ in the fiber length of 5 meters[8]; the special resolution of $4 \mathrm{~m}$ is obtained with frequency sweeping, but it only gives the frequency change without absolute amplitude[9]; To the existing OTDR measuring system with UWFBGs, the wavelength resolution of $9.03 \mathrm{pm}$ and static strain resolution of $4.73 \mathrm{u} \varepsilon$ can be achieved by using a dispersive unit to convert the wavelength shift into time-delay changes, and dispersive component is easy to be disturbed[6-7]; it can measure temperature with maximum deviation of $1.69^{\circ} \mathrm{C}$ and average error of $0.56^{\circ} \mathrm{C}$ by obtaining the intensity of reflection light, however the light intensity is not a stable factor[10]; The temperature resolution of $0.11^{\circ} \mathrm{C}$ is achieved by optical route and CCD electric circuit, but the whole system is complex and expensive[11]. From all listed above, it is shown that 
the measuring accuracy, fiber length and system stability are all need to be improved.

In this paper, the distributed OTDR system with UWFBGs based on correlation degree spectrum is proposed. The reflection spectrum is the intrinsic features of UWFBGs, except the central wavelength and reflection intensity, other features aren't be effected by outer physical factors. Meanwhile, the structure of the OTDR system in this paper only adds one tunable filter contrast with traditional OTDR system[12-13], and only the relative parameters are needed during the measuring procedure. Hence, the measuring system based on this technique is more stable than other methods using special optical components[6-7]. Moreover, the phase-OTDR systems with UWFBGs use the narrow line-width laser, there must be undesirable noise caused by interference among UWFBGs, which will eliminate the SNR of whole system. This view is discussed in section 2, and it hasn't seen the report about the comparison of SNR in 2 kinds of OTDR system with UWFBGs. Furthermore, with the curve fitting and the correlation degree spectrum algorithm, the improvement of SNR of reflection spectrum and the distributed multiple physical factors measurement are achieved.

Define the correlation degree spectrum and explain the advantage of correlation degree spectrum over other technique). Explain how this principle is explored. Why it works better than phase OTDR.

Since you are reporting temperature and strain measurement, explain your accuracy comparting with reported phase OTDR accuracy for temperature and strain measurement, as well as number of FBGs, and spatial resolution.

The rest of the paper is organized as below: Section II describes the new distributed OTDR measuring system with tunable filter, the spectrum features of UWFBGs and gives the define of correlation degree spectrum(CDS). CW noise signals and SNR inner the UWFBGs is verified by experiment in section III. Section IV gives the performance analysis of the OTDR system with CDS. Section $\mathrm{V}$ is the conclusion.

\section{Principle of correlation degree spectrum}

\section{Reflection spectrum of UWFBGs}

The reflection spectrum sweeping experimental system is shown in Fig 1a. The $-3 \mathrm{~dB}$ bandwidth of broad band source is $2 \mathrm{~nm}$, and AFG coordinates EOM to modulate the broad band light to generate the pulse, whose width is $8 \mathrm{~ns}$ and repetition is $1 \mathrm{KHz}$. The length of UWFBGs is $810 \mathrm{~m}$, reflectivity is $0.1 \%$ and the interval between 2 UWFBGs is $2.5 \mathrm{~m}$. A TF is used at the receive end, and its wavelength resolution is $3.2 \mathrm{pm} / \mathrm{mV}$. The central wavelengths of TF can be set in different wavelength within the range of reflection spectrum of UWFBGs, and the different wavelength position can cause different reflection intensity of UWFBGs, as shown in Fig 1b. Assume the central wavelength of the TF is set at $1551.168 \mathrm{~nm}$, once the UWFBG is affected by strain or temperature around, its bragg wavelength or reflection spectrum will shift within the range of $2 \mathrm{~nm}$, thence the intensity of UWFBG reflection signal outputting from TF will change consequently. Therefore, the whole reflection spectrum of every UWFBG in the same fiber can be obtained by using TF to sweep the range of broad band light source, through changing the voltage added on the TF consecutively. 


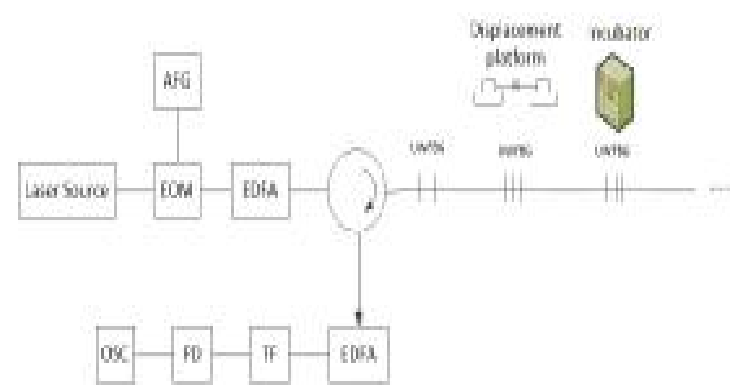

(a)

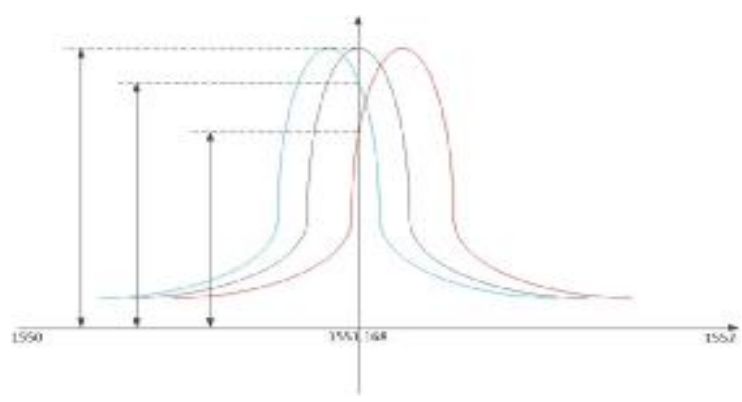

(b)

Fig.1 Structure and principle of experimental system. (a)Structure diagram of experimental system. PC: Polarization Controller. PBS: Polarization Beam Splitter. EOM: Electronic Optical Modulator. AFG: Arbitrary Function Generator. TF: Tunable Filter. PD: Photon Detector. OSC: Oscilloscope.(b) Principle of spectrum sweeping

Arbitrary 10 series connecting UWFBGs are selected in the sweeping experiment. The spectrum profiles of these 10 UWFBGs are revealed in Fig 2. Fig 2a is the real shape of the 10 reflection spectrum profiles, and Fig $2 b$ is the shape after being normalized. The correlation degree of the 10 normalized reflection spectrum profiles are listed in Table 1. Choosing one spectrum profile as the reference, the other 9 ones are made correlation with the reference, then the worst one among the 9 correlation degree results is $99.34 \%$. Hereafter, the fitting curve(black line) can be seen in Fig $6 \mathrm{~b}$ also, its function is Eq 1. Above the normalization amplitude 0.2, the correlation degree between Eq 6 and spectrum profiles exceeds $99.5 \%$. Therefore, the spectrum profile of the total UWFBGs in one fiber can be considered the same in statistics. Eq 1 is used as the function of normalized reflection spectrum profile in this paper, the parameter $a$ and $b$ are changed according to the spectrum and $\lambda_{0}$ is the central wavelength.

$$
a \exp \left[-\left(\lambda-\lambda_{0}\right) / b\right]^{2} \quad \lambda \in(1550.168 n m, 1552.168 n m)
$$

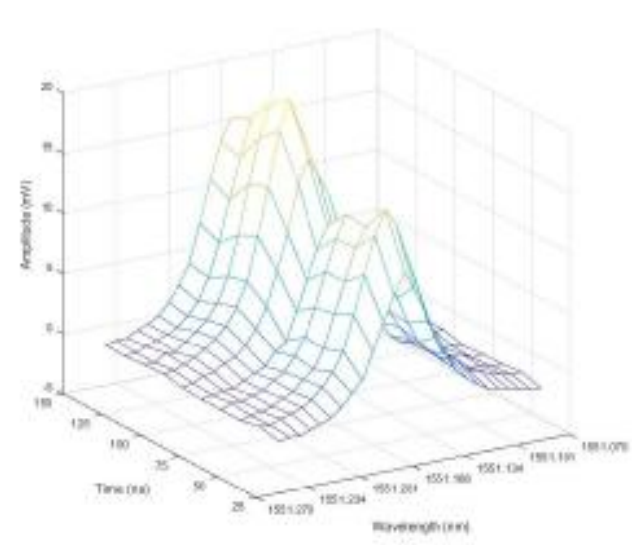

(a)

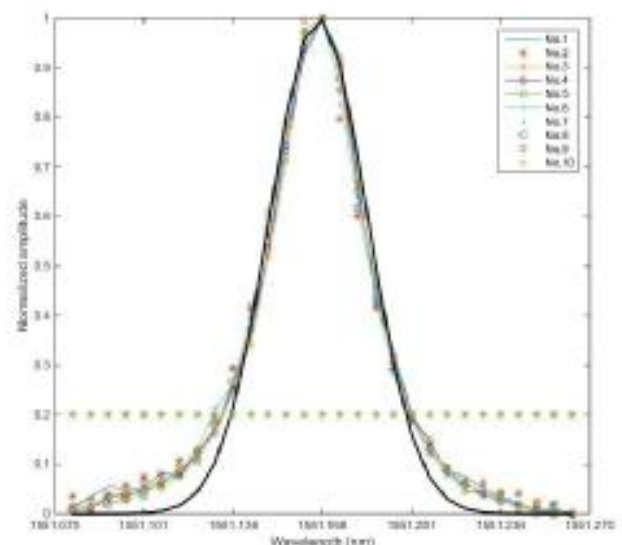

(b)

Fig.2 Spectrum profile of the 10 UWFBGs

Table 1 Correlation degree among the 10 UWFBGs

\begin{tabular}{|l|l|l|l|l|l|l|l|l|}
\hline 1 to ref & 2 to ref & 3 to ref & 4 to ref & 5 to ref & 6 to ref & 7 to ref & 8 to ref & 9 to ref \\
\hline $99.34 \%$ & $99.8 \%$ & $99.88 \%$ & $99.83 \%$ & $99.61 \%$ & $99.74 \%$ & $99.78 \%$ & $99.44 \%$ & $99.86 \%$ \\
\hline
\end{tabular}

Curve fitting

The TF is driven by periodic continuous voltage, and its central wavelength can sweep the whole sensing range back and forth. That's to say, every time the TF sweeps the sensing range, and find the peak intensity of UWFBGs at different positions, then the spectrum profile of all UWFBGs can be obtained and fitting them according to $\mathrm{Eq}(6)$. Therefore, the correlation degree between the spectrum profile of any UWFBG at time $t$ and $t-1$ can be calculated, and the change of correlation 
degree will flow the change of the physical factors being measured.

The sweeping result of one UWFBG is displayed in Fig 3, at the position where wavelength is $1551.26 \mathrm{~nm}$, the spectrum profile has been swept 5 times and every dot in the spectrum is averaged 20 times. The black curve also is the fitting curve according to $\operatorname{Eq}(1)$, and red line has the same meaning as the green star line in Fig $2 \mathrm{~b}$. The SNR(signal-to-noise ratio) of the reflection spectrum after fitting is calculated according to $\mathrm{Eq}(2)$, I expresses the vector of signal after fitting, $I_{N}$ expresses the vector of raw signal after removing noise. Thence the SNR of reflection spectrum over $35 \mathrm{~dB}$ is achieved. That means the measured displacement/ strain cannot be smaller than $3.2 \mathrm{pm} / 3.2 u \xi$.

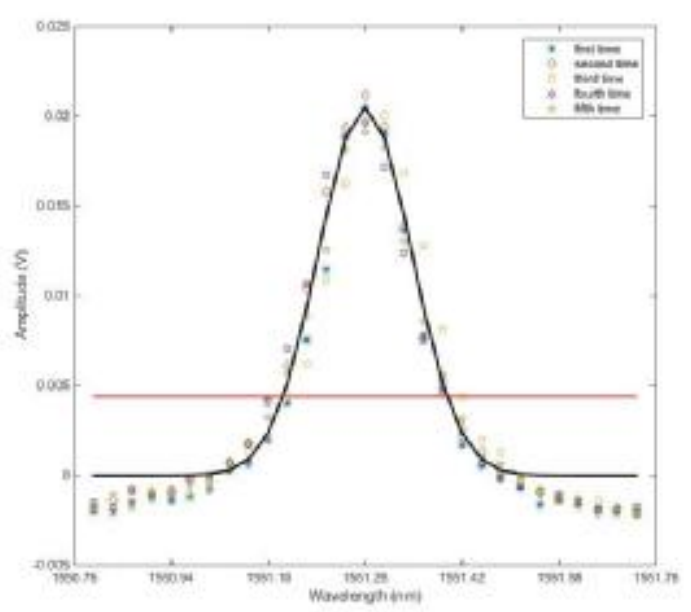

Fig.3 Fitting curve of spectrum profile

$$
\left\{\begin{array}{l}
V_{\text {signal }}=\left(\sum I^{2}\right) / \operatorname{Length}(I) \\
V_{\text {noise }}=\left(\sum\left(I-I_{N}\right)^{2}\right) / \operatorname{Length}(I) \\
S N R=20 \lg \left(V_{\text {signal }} / V_{\text {noise }}\right)
\end{array}\right.
$$

\section{Correlation degree spectrum}

The correlation degree spectrum(CDS) is defined as the root of the overlapping area of two spectrum as the shadow part shown in Fig.4. The spectrum is after fitting and normalized, and only the parts above 0.2 are needed. When no spectrum shift is generated(at original spectrum position), the CDS is maximum 6.73. When spectrum shift is caused by strain or temperature, the CDS decreases(at spectrum shift position1), until it equals 0(at spectrum shift position2). Therefore, the change of the strain or temperature is bigger, the value of CDS is smaller.

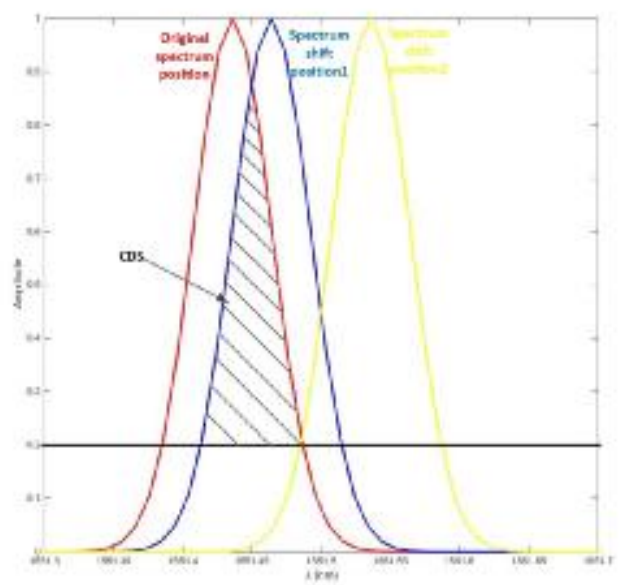

Fig.4 Correlation degree spectrum 


\section{CW noise and SNR}

In order to analyze quantitatively the performance of phase-OTDR and OTDR system with UWFBGs, the structure of experimental system is used shown in Fig. 1a. In phase-OTDR system, a laser source with linewidth of $200 \mathrm{Khz}$ (New Focus TLB-6600) is used. However, a broad band laser source $(-3 \mathrm{~dB}$ bandwidth is $2 \mathrm{~nm})$ is utilized in OTDR system. The experimental results of CW noise in phase-OTDR system and OTDR system both with UWFBGs are shown in Fig 5. The red line is synchronous signal of pulse, blue line is the trace of UWFBGs. In Fig.5a, suffered by the intrinsic interference, the CW noise in phase-OTDR trace can over $1 \mathrm{v}$, which nearly equals the amplitude of UWFBGs signal it selves, and the SNR of the trace is about $-2.49 \mathrm{~dB}$. However, the CW noise in OTDR trace shown in Fig.5b is only $250 \mathrm{mv}$ approximately, and the SNR of the trace is nearly $13.6 \mathrm{~dB}$. Therefore, once the number of UWFBGs is large, the phase-OTDR system with UWFBGs cannot work regularly.

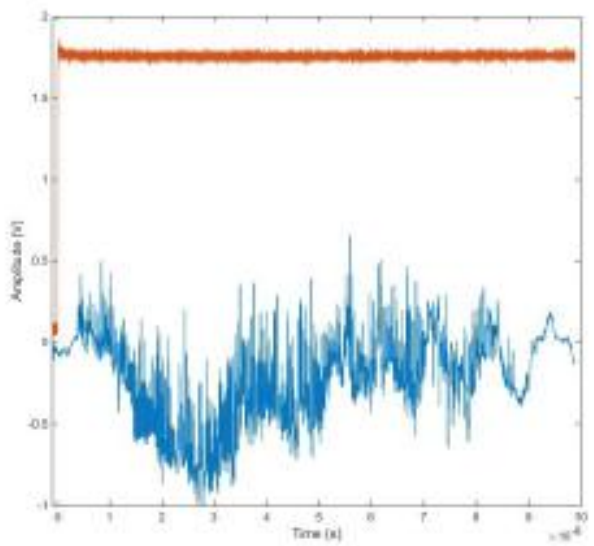

(a)

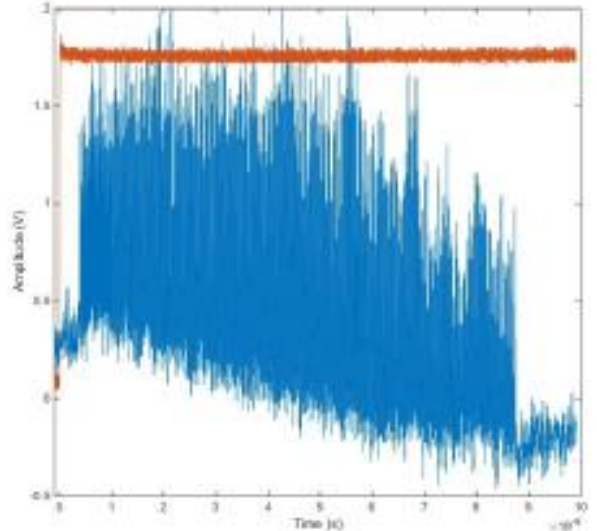

(b)

Fig.5 Trace in different experimental system. (a)Phase-OTDR. (b) OTDR

Under different peak power of injected pulse, the energy fluctuations in the whole trace are shown in Fig.6. $0 \mathrm{~m}$ means the begin of the fiber, $810 \mathrm{~m}$ means the end of the fiber. Within the fiber, there are 3 fixed positions of $120 \mathrm{~m}, 320 \mathrm{~m}$ and $622.5 \mathrm{~m}$ separately. The traces of OTDR system with UWFBGs are changed in the same trend with the peak power change of injected pulse, however the trace fluctuations of phase-OTDR system with UWFBGs are very disordered because of the inner intrinsic interference.
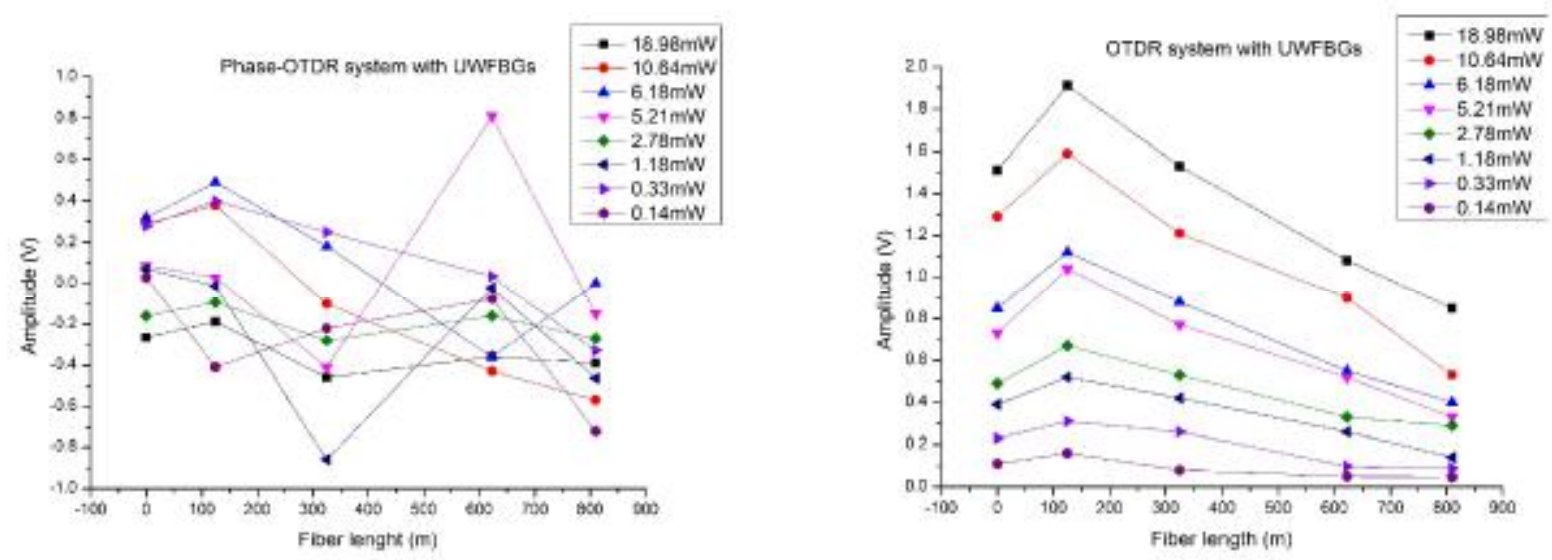

Fig.6 Fluctuation at different position in the whole trace.(a)Trace change of Phase-OTDR in different injected power.(b)Trace change of OTDR in different injected power 


\section{Performance analysis}

Sensing range and Spatial resolution

The $-3 \mathrm{~dB}$ and $-6 \mathrm{~dB}$ bandwidth of the broad band source are $2 \mathrm{~nm}$ and $4.5 \mathrm{~nm}$ separately, it means the range of the reflection spectrum of one UWFBG is within $4.5 \mathrm{~nm}$. Meanwhile, the distance from the position of the reflection spectrum to the edge of the broad band light spectrum is shorter, the intensity of the reflection light is smaller.

Spatial resolution is mainly decided by the pulse width, and the extreme condition that two adjacent UWFBGs experience opposite change of physical factors must be considered. The minimum interval $L$ between two adjacent UWFBGs should be chosen to avoid overlap, and it is expressed as:

$$
L \geq v_{g} \tau / 2+2 \Delta \lambda_{\max }
$$

In $\mathrm{Eq}(3), v_{g}$ is the group velocity in fiber, $\tau$ is pulse width, $\Delta \lambda_{\max }$ is the maximum wavelength

shift of UWFBG.

Strain and Temperature response

The displacement platform shown in Fig 1a is used to generate strain to UWFBG. The normalized spectrum profiles corresponding to strain added to UWFBG from $50-250 u \xi$ are displayed in Fig 7a, furthermore the spectrum profile meets the $\operatorname{Eq}(1)$, and the linear relationship between strain and correlation degree of spectrum profile is shown in Fig 7b. Only the area whose normalized amplitude higher than 0.2 is needed in one spectrum profile, any overlapping area below black amplitude line of 0.2 has no meanings. That's why the line in Fig $7 \mathrm{~b}$ is discontinuous. A whole measuring procedure is made up of two turns. Specifically, in Fig 7a, the red spectrum profile is the original that without effect of strain, the other spectrum profiles with different colors indicate the procedure of wavelength shifting caused by strain. The overlapping areas among red and blue and green and black spectrum profiles are over the black amplitude line of 0.2 , they belong to the first turn. However the overlapping area between red and yellow spectrum profiles is below the black amplitude line of 0.2 , it belongs to the second turn. When the spectrum profile shifts to one position, where it's overlapping area with original red profile is zero, the first measuring turn is finished and the second turn begins, meanwhile it corresponds to the position of line hopping in Fig $7 \mathrm{~b}$.

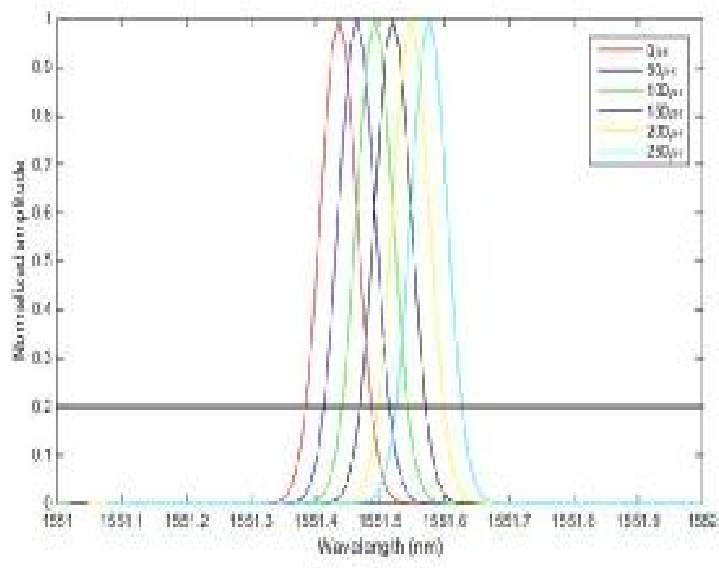

a Spectrum profiles

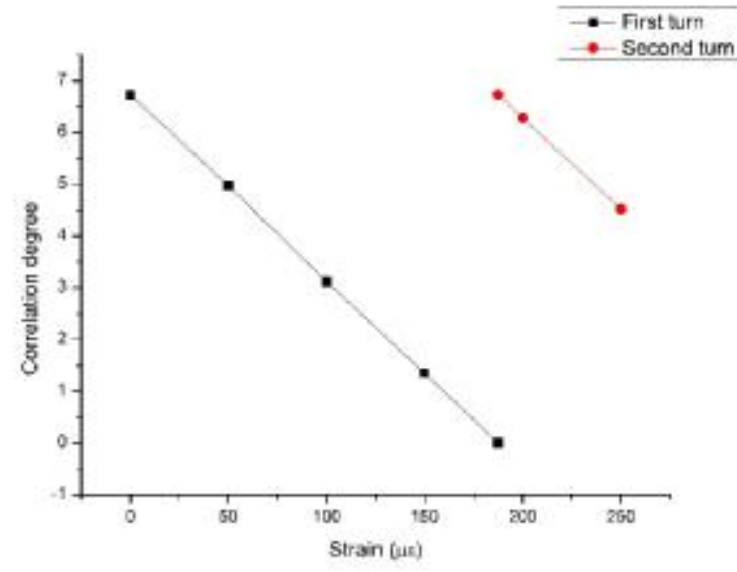

b Relationship between strain and correlation degree spectrum

Fig.7 Strain measurement

When there is no strain is measured, the correlation degree is the maximum value 6.73. At the end of the first measuring turn, the measured strain is $187 u \xi$, and the correlation degree is zero. In 
the sensing system, the CDS-strain coefficient is $0.116 / 3.2 u \xi$.

Fig 8 shows the results of changing the peak power of pulse, when the strain changes from $200 u \xi$ to $250 u \xi$. It can be known that the amount of wavelength change and fluctuation of trace will be affected by peak power of pulse, that's to say the suitable power of pulse should be chosen to increase the signal-to-noise ratio of the sensing system.

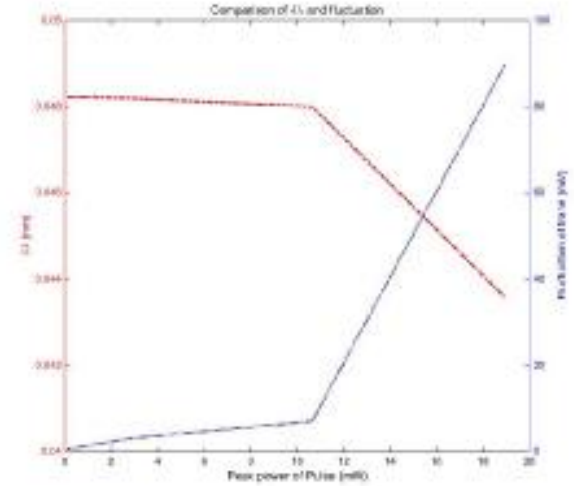

Fig. 8 Effect of different peak power of pulse

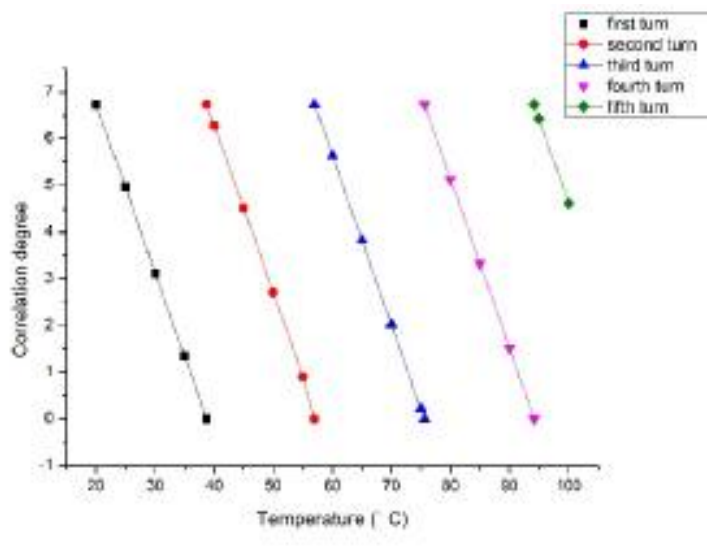

Fig. 9 Result of sensing temperature

On the other hand, the temperature sensing experiment is done in an incubator, the temperature is changed from 20 to $100^{\circ} \mathrm{C}$, and the sensing result is shown in Fig 9. The whole sensing procedure is made up of 5 turns, and the temperature resolution is $0.32^{\circ} \mathrm{C}$.

\section{Conclusion}

In summary, a distributed sensing system based on UWFBG's spectrum profile is depicted and verified by experiment. In the sensing procedure, only the relative change of two spectrum profile is needed, no absolute value is sensed. Therefore, the stability of the sensing system is reliable. Its sensing span is decided by the broad band light source, and the spatial resolution is depending on the pulse width and changing range of sensed physical factors. The accuracy of measuring strain and temperature can achieve $3.2 u \xi$ and $0.32^{\circ} \mathrm{C}$, separately.

\section{References}

[1] X. Li, Q. Sun, J. Wo, M. Zhang, and D. Liu, "Hybrid TDM/WDM-based fiber-optic sensor network for perimeter intrusion detection," J. Lightw. Technol., vol. 30, no. 8, pp. 1113-1120, Apr. 15, (2012).

[2] H. Iida, Y. Koshikiya, F. Ito, and K. Tanaka, "High-sensitivity coherent optical time domain reflectometry employing frequency-division multiplexing," J. Lightwave Technol. 30(8), 1121-1126 (2012).

[3] J. Van Der Horst, J. L. Lopez, W. Berlang, and H. Potters, "In-well distributed fiber optic solutions for reservoir surveillance," in Proc. Offshore Technol. Conf., (2013).

[4] Chen Wang, Ying Shang, Xiao-Hui Liu, Chang Wang, Hai-Hu Yu, De-Sheng Jiang, and Gang-Ding Peng. Distributed OTDR-interferometric sensing network with identical ultra-weak fiber Bragg gratings, 2 Nov (2015), Vol. 23, No. 22, DOI:10.1364/OE.23.029038, OPTICS EXPRESS 29038.

[5] Chen Wang, Ying Shang, Xiaohui Liu, Chang Wang and Gangding Peng, Distributed acoustic mapping based on self-interferometry of phase-OTDR, Asia Communications \& Photonics 
Conference, (2014) : ATh3A.213.

[6] L. Ma, C. Ma, Y. Wang, D.Y. Wang and A. Wang. High-speed quasi-distributed strain sensing based on time division multiplexing of fibre Bragg gratings, ELECTRONICS LETTERS 14th April (2016), Vol. 52 No. 8 pp. 643-645

[7] Lingmei Ma, Cheng Ma, Yunmiao Wang, Dorothy Y. Wang, and Anbo Wang. High-Speed Distributed Sensing Based on Ultra Weak FBGs and Chromatic Dispersion, IEEE PHOTONICS TECHNOLOGY LETTERS, VOL. 28, NO. 12, JUNE 15, (2016) 1344-1347

[8] AMELIA LAVINIA RICCHIUTI AND SALVADOR SALES. Spot event detection along a large-scale sensor based on ultra-weak fiber Bragg gratings using time-frequency,Vol. 55, No. 5 / February 10 (2016). Applied Optics analysis, 1054-1060, dio:10.1364/AO.55.001054.

[9] Xuping Zhang, Zheng Guo, Yuanyuan Shan, Zhenhong Sun, Siyi Fu, Yixin Zhang, "Enhanced $\Phi-O T D R$ system for quantitative strain measurement based on ultra-weak fiber Bragg grating array,” Opt. Eng. 55(5), 054103 (2016), doi: 10.1117/1.OE.55.5.054103.

[10] M. Zhang et al., "A large capacity sensing network with identical weak fiber Bragg gratings multiplexing," Opt. Comm. vol. 285, no. 13/14, pp. 3082-3087, Jun, (2012).

[11] C. Hu, H. Wen, and W. Bai, "A novel interrogation system for large scale sensing network with identical ultra-weak fiber Bragg gratings,” J. Lightwave Technol. 32, 1406-1411 (2014).

[12] A Masoudi, M Belal and T P Newson. A distributed optical fibre dynamic strain sensor based on phase-OTDR, MEASUREMENT SCIENCE AND TECHNOLOGY, 24 (2013) 085204 (7pp) doi:10.1088/0957-0233/24/8/085204.

[13] Gaosheng Fang, Tuanwei Xu, Shengwen Feng, and Fang Li, Phase-Sensitive Optical Time Domain Reflectometer Based on Phase-Generated Carrier Algorithm, JOURNAL OF LIGHTWAVE TECHNOLOGY, VOL. 33, NO. 13, JULY 1, (2015), pp.2811-16. 